\title{
Resilience and Vulnerability:
}

\section{Prolonged Grief in the Bereaved Spouses of Marital Partners who Died of AIDS}

\author{
Nancy Xiaonan $\mathrm{Yu}^{*}$ \\ Department of Applied Social Sciences, City University of Hong Kong, Hong Kong, China \\ Cecilia L. W. Chan \\ Department of Social Work and Social Administration and Center on Behavioral Health, \\ The University of Hong Kong, Hong Kong, China \\ Jianxin Zhang \\ Institute of Psychology, Chinese Academy of Sciences, Beijing, China \\ Sunita M. Stewart \\ Department of Psychiatry, University of Texas Southwestern Medical Center at Dallas, TX, USA
}

\footnotetext{
* Corresponding author. Department of Applied Social Sciences, City University of Hong Kong, Tat Chee Avenue, Kowloon, HKSAR, China. Tel: +852 34429436. Fax: +852 34420283. E-mail: nancy.yu@ @ityu.edu.hk.
} 


\begin{abstract}
Spousal bereavement is closely linked to prolonged grief, i.e. significant adjustment symptoms that last for more than six months after the loss. This article focused on potential risk and protective factors that may influence bereavement outcomes. Participants in this study were surviving spouses of individuals who died of AIDS. These participants were themselves living with HIV. In this cross-sectional study, 120 bereaved participants completed measures of grief, quality of dying and death of the deceased, negative conceptions of death resulting from AIDS, death attitudes, and personal resilience. The results showed that one third $(35.0 \%)$ of the bereaved participants reported grief levels above the prolonged grief cut off scores, and can be categorized as the "prolonged grief" group. Although quality of dying and death was not associated with the intensity of grief, negative conceptions of death from AIDS, fear of death and resilience independently predicted grief symptoms in the regression models. Our findings provide insight into the grief process for the surviving spouse of AIDS victims in rural China. Since resilience is malleable, developing resilience interventions to enhance adjustment to bereavement may be a promising direction in grief counselling and therapies.
\end{abstract}

Keywords: Prolonged grief, quality of dying and death, conceptions of death, death attitudes, resilience, HIV, AIDS 
Bereavement due to death of a spouse has been described as one of the most stressful life events (Prigerson et al., 1995). Prolonged grief that continues for more than six months after the loss is a persistent and debilitating psychological phenomenon that includes searching for the deceased, yearning, preoccupation, disbelief about the death, avoidance of reminders, hallucinations, bitterness, and survivor guilt (Prigerson et al., 1996), and is associated with many health risks. The rural population of people living with HIV (PLHIV) in China is understudied. We examined risk and protective factors associated with grief symptoms in the surviving spouse of an individual who died of acquired immune deficiency syndrome (AIDS) (Prigerson et al., 1997).

We examined the following possible contributors: Higher quality of dying and death, including pain control, death preparation, connection with the family and social groups, and transcendence (e.g., being unafraid of death, being unworried about strain on loved ones) (Downey et al., 2010), may make it easier for the bereaved to accept the death and experience fewer negative symptoms. Negative conceptions of HIV+ individuals and their death are prevalent in many cultures (e.g., Niehaus, 2007) including China (Cao et al., 2006; Chen et al., 2011), and may interfere with adaptation of the bereaved. In conceptualizing the process of loss, Neimeyer et al. (2003) proposed that negative as well as positive death attitudes, including fear, avoidance, and acceptance, might influence the reconstruction of meaning of death in the bereaved and thereby facilitate or disrupt the natural process of mourning. Resilience, defined as a general tendency to quickly recover from negative life events, has been documented to help individuals cope with various traumatic events (Wagnild \& Young, 1993). There has been a greater interest in positive coping strategies among those who have lost a loved one to AIDS (Rogers, Hansen, Levy, Tate, \& Sikkema, 2005). Other investigators have proposed that resilience in the HIV+ context may allow individuals to regain equilibrium because of their optimism about their ability to manage challenges in life, ability to activate strengths and 
resources including support networks of family and friends, and flexibility in adjusting to the loss (e.g., Bletzer, 2007; Delport, Strydom, Theron, \& Geyer, 2011; Farber, Schwartz, Schaper, Moonen, \& McDaniel, 2000; Sikkema et al., 2000). We hypothesized that in bereaved spousal survivors assessed six or more months after their loss: intensity of grief symptoms would be negatively associated with quality of dying and death of the deceased and resilience, and positively associated with negative conceptions of death from AIDS and negative death attitudes.

\section{Methods}

\section{Participants}

A convenience sample of 120 participants from a randomly selected seven of 22 villages in central China with HIV prevalence greater than $10 \%$ was enrolled in this study. Impoverished villagers in rural China who contracted HIV as a result of commercial blood transactions, often transmitted the disease to their partners (Wu et al., 1995). These surviving partners face a double set of stressors: the death of the spouse, and their own mortality. Participants were HIV+ and had a spouse who died from AIDS between six and 36 months prior to the study. This timeframe is referenced to the range of period since bereavement in previous studies (Prigerson et al., 1996; Schwartzberg \& Janoff-Bulman, 1991). They were referred to the research staff by healthcare providers who monitored their medication (Highly Active Antiretroviral Therapy, response rate $=80.0 \%$ ). Trained interviewers conducted face-to-face interviews in a private setting. Participants received monetary compensation for their time spent on this survey (CNY30, approximately equivalent to US \$5). Participants provided written informed consent before the study commenced. Ethics approval was obtained from the Ethical Review Board of the City University of Hong Kong.

\section{Measures}

Grief was measured by the 19 item Inventory of Complicated Grief (ICG; Prigerson, et al., 1995), which describes bereavement-related thoughts and behaviors (e.g., "preoccupation 
with the person who died"). A score of 30 has been suggested as cutoff for high risk for lossrelated impairment in functioning (Shear, Jackson, Essock, Donahue, \& Felton, 2006). This scale has been used to measure prolonged grief in the Chinese population (Chiu et al., 2010; Xu et al., 2014). Cronbach'salpha $(\alpha)$ was .88 in the present study.

Quality of dying and death of the deceased spouse was measured by the single item Quality of Dying and Death Questionnaire (Downey et al., 2010): “Overall, how would you rate the quality of your spouse's dying and death?", rated on an 11-point scale, with high scores indicating better quality.

Negative conceptions of death of AIDS of the bereaved were measured by five items developed by the researchers based on interviews with AIDS bereaved family members. Participants were asked to compare the death from AIDS with those from other illnesses such as cancer and coronary heart disease (e.g., "AIDS patients die with less dignity") on a 5-item scale; higher scores indicated worse conceptions. The five items loaded onto a single factor, explaining $59.37 \%$ of the variance $(\alpha=.88)$.

Death attitudes were measured by the 32 item Death Attitude Profile-revised (Wong et al., 1994), which has a validated Chinese version (A.H.Y. Ho et al., 2010). In the present study, five of the seven subscales (approach acceptance, fear of death, death avoidance, escape acceptance, and personal acceptance) which had acceptable or better reliability ( $\alpha$ 's $=.65$ to .94$)$ were used.

Resilience was measured by the 25 item Connor-Davidson Resilience Scale (CD-RISC) (Connor \& Davidson, 2003). This scale has been used to measure resilience in another Chinese HIV+ sample (Yu et al., 2009) $(\alpha=.96)$.

\section{Results}

Table 1 provides the demographics for the sample. Forty two (35.0\%) fell into the “prolonged grief” category (ICG scores of 30 or above). 
The intensity of grief symptoms was positively associated with negative conceptions of death from AIDS (Pearson's $r=.22, p<.05)$, and death attitudes, specifically fear of death $(r$ $=.38, p<.01)$ and escape acceptance $(r=.22 . p<.05)$; and they were negatively associated with resilience $(r=-.25, p<.05)$. In the regression model, after controlling for demographic variables which accounted for $17 \%$ of the variance in grief symptoms, negative conceptions of death from $\operatorname{AIDS}(\beta=.19, p=.04)$, fear of death $(\beta=.40, p<.001)$, and resilience $(\beta=-.29, p=.003)$ independently added to the prediction of grief symptoms. Sex did not moderate these results.

\section{Discussion}

One third of our sample reported symptoms above the cutoff score indicating prolonged grief. Contrary to our hypothesis, grief symptoms were not associated with quality of dying and death in the present study. Prolonged grief may be less influenced by the acute end-of-life experience, but rather by attachment vulnerabilities and the quality of the long-term relationship (Shear \& Shair, 2005).

Our findings on the association of grief symptoms and negative conceptions of death are novel. Negative conceptions of AIDS-related death may heighten anxiety about these participants' own death, and may be specific to survivors who themselves can anticipate a similar death. Fear of death was the only attitude dimension that predicted grief symptoms in the regression models. Negative beliefs about death and the afterlife are abundant in Chinese culture (Chan et al., 2005). In the context of the meaning-making framework of Currier et al. (2006), the challenge for clinicians working with this group is how to understand grief, loss, and stresses associated with HIV/AIDS, in a manner that reconstructs the entirely negative meaning of death into something that can make sense to this bereaved population.

Our findings replicate and extend previous research on the protective effects of resilience into a new context. Low levels of resilience might prospectively identify bereaved individuals who are at risk for prolonged grief. Of particular importance is that resilience can be effectively 
enhanced at a low cost, not only in individuals in North America (Steinhardt \& Dolbier, 2008), but also in rural Chinese PLHIV infected via plasma donation (Yu et al., 2014). A psychological intervention with a broader framework that includes elements of effective coping and resilience (e.g., positive thinking, self-efficacy, emotion regulation) may be a promising direction in grief counseling and therapies (Lin et al., 2004).

The cross-sectional design of this study limits directional hypotheses, and prolonged grief following bereavement may result in lower resilience. A prospective, longitudinal design would contribute significantly to the understanding prolonged grief in this population. The self-report and retrospective methods used may be subject to bias. Quality of dying and death was assessed using a single item. Convenience sampling may have resulted in participants with unrepresentative levels of grief (Babbie, 2013).

Despite these limitations, by identifying its association with negative conceptions and fear of death in the bereaved, the current investigation adds to the growing understanding of the concomitants of prolonged grief in the understudied group of surviving HIV+ spouses of those who die from AIDS in China.

\section{Role of the Funding Source}

This study was supported by College Research Grant, College of Liberal Arts and Social Sciences, City University of Hong Kong. The sponsor had no further role in study design, in the collection, analysis and interpretation of data, in the writing of the report, and in the decision to submit the paper for publication. 


\section{References}

Babbie, E. R. (2013). The practice of social research. Belmont, CA: Thomson Wadsworth.

Bletzer, K. V. (2007). Identity and resilience among persons with HIV: A rural African American experience. Qual Health Res, 17(2), 162-175.

Cao, X., Sullivan, S.G., Xu, J., \& Wu, Z. (2006). Understanding HIV-related stigma and discrimination in a "blameless" population. AIDS Education and Prevention, 18, 518-528.

Chan, C.L.W., Chow, A.Y.M., Ho, S.M.Y., Tsui, Y.K.Y., Tin, A.F., Koo, B.W.K., \& Koo, E.W.K., (2005). The experience of Chinese bereaved persons: A preliminary study of meaning making and continuing bonds. Death Studies, 29, 923-947.

Chiu, Y.W., Huang, C.T., Yin, S.M., Huang, Y.C., Chien, C.H., \& Chuang, H.Y. (2010).

Determinants of complicated grief in caregivers who cared for terminal cancer patients. Supportive Care in Cancer, 18, 1321-1327.

Connor, K.M., \& Davidson, J.R. (2003). Development of a new resilience scale: The ConnorDavidson Resilience Scale (CD-RISC). Depression and Anxiety, 18, 76-82.

Currier, J.M., Holland, J.M., \& Neimeyer, R.A. (2006). Sense-making, grief, and the experience of violent loss: Toward a mediational model. Death Studies, 30, 403-428.

Delport, R., Strydom, H., Theron, L., \& Geyer, S. (2011). Voices of HIV\&AIDS-affected educators: How they are psychosocially affected and how REds enabled their resilience. AIDS Care, 23(1), 121-126.

Downey, L., Curtis, J.R., Lafferty, W.E., Herting, J.R., \& Engelberg, R.A. (2010). The Quality of Dying and Death Questionnaire (QODD): Empirical domains and theoretical perspectives. Journal of Pain and Symptom Management, 39, 9-22.

Farber, E. W., Schwartz, J. A. J., Schaper, P. E., Moonen, D. J., \& McDaniel, J. S. (2000). Resilience factors associated with adaptation to HIV disease. Psychosomatics, 41(2), 140146.

Hansen, N.B., Tarakeshwar, N., Ghebremichael, M., Zhang, H., Kochman, A., \& Sikkema, K.J. (2006. Longitudinal effects of coping on outcome in a randomized controlled trial of a group intervention for HIV-positive adults with AIDS-related bereavement. Death Studies, 30, 609-636.

Ho, A.H.Y., Chan, C.L.W., Chow, A.Y.M., Pon, A.K.L., \& Ng, S.M. (2010). Psychometric properties of the Chinese version (C-DAP-R) of the Death Attitude Profile-Revised. Illness, Crisis, \& Loss, 18, 95-110. 
Lin, K.K., Sandler, I.N., Ayers, T.S., Wolchik, S.A., \& Luecken, L.J. (2004). Resilience in parentally bereaved children and adolescents seeking preventive services. Journal of Clinical Child \& Adolescent Psychology, 33, 673-683.

Neimeyer, R.A., Moser, R.P., \& Wittkowski, J. (2003). Assessing attitudes toward dying and death: Psychometric considerations. Omega: Journal of Death and Dying, 47, 45-76.

Niehaus, I. (2007). Death before dying: Understanding AIDS stigma in the South African Lowveld. Journal of Southern African Studies, 33(4), 845-860.

Prigerson, H.G., Bierhals, A.J., Kasl, S.V., Reynolds, C.F., \& Jacobs, S.C. (1996). Complicated grief as a distinct disorder from bereavement-related depression and anxiety: A replication study. American Journal of Psychiatry, 153, 1484-1486.

Prigerson, H.G., Maciejewski, P.K., Reyolds, C.F., Bierhals, A.J., Newsom, J.T., Fasiczka, A., Frank, E., Doman, J., Miller, M. (1995). Inventory of Complicated Grief: A scale to measure maladaptive symptoms of loss. Psychiatry Research, 59, 65-79.

Prigerson, H.G., Shear, M.K., Frank, E., Beery, L.C., Silberman, R., Prigerson, J., \& Reynolds, C.F. (1997). Traumatic grief: A case of loss-induced trauma. American Journal of Psychiatry, 154, 1-5.

Rogers, M. E., Hansen, N. B., Levy, B. R., Tate, D. C., \& Sikkema, K. J. (2005). Optimism and coping with loss in bereaved HIV-infected men and women. Journal of Social and Clinical Psychology, 24(3), 341-360.

Schwartzberg, S. S., \& Janoff-Bulman, R. (1991). Grief and the search for meaning: Exploring the assumptive worlds of bereaved college students. Journal of Social and Clinical Psychology, 10(3), 270-288.

Shear, K., \& Shair, H. (2005). Attachment, loss, and complicated grief. Developmental Psychobiology, 47(3), 253-267.

Shear, K.M., Jackson, C.T., Essock, S.M., Donahue, S.A., \& Felton, C.J. (2006). Screening for complicated grief among Project Liberty service recipients 18 months after September 11, 2001. Psychiatry Service, 57, 1291-1297.

Sikkema, K. J., Kalichman, S. C., Hoffmann, R., Koob, J. J., Kelly, J. A., \& Heckman, T. G. (2000). Coping strategies and emotional wellbeing among HIV-infected men and women experiencing AIDS-related bereavement. AIDS Care, 12(5), 613-624.

Steinhardt, M., \& Dolbier, C. (2008). Evaluation of a resilience intervention to enhance coping strategies and protective factors and decrease symptomatology. Journal of American College Health, 56, 445-453. 
Wagnild, G.M., \& Young, H.M. (1993). Development and psychometric evaluation of the Resilience Scale. Journal of Nursing Measurement, 1, 165-178.

Wong, P.T.P., Reker, G.T., \& Gesser, G. (1994). Death Attitude Profile-Revised: A multidimensional measure of attitudes toward death in Neimeyer, R.A. (Ed.), Death anxiety handbook: Research, instrumentation, and application. Taylor and Francis, Washington, DC, pp. 121-148.

Wu, Z., Liu, Z., \& Detels, R. (1995). HIV-1 infection in commercial plasma donors in China. Lancet, 346, 61-62.

Xu, Y., Herrman, H., Bentley, R., Tsutsumi, A., \& Fisher, J. (2014). Effect of having a subsequent child on the mental health of women who lost a child in the 2008 Sichuan earthquake: A cross-sectional study. Bull World Health Organ, 92, 348-355.

Yu, X.N., Lau, J.T.F., Mak, W.W.S., Cheng, Y., Lv, Y., \& Zhang, J. (2014). A pilot theory-based intervention to improve resilience, psycho-social well-being and quality of life among people living with HIV in rural China. Journal of Sex \& Marital Therapy, 40, 1-16.

Yu, X.N., Lau, J.T.F., Mak, W.W.S., Cheng, Y.M., Lv, Y.H., \& Zhang, J.X. (2009). Risk and protective factors in association with mental health problems among people living with HIV who were former plasma/blood donors in rural China. AIDS Care, 21, 645-654. 
Table 1. Demographic characteristics of the participants

$$
\text { n (\%) or }
$$

Mean \pm SD

\section{$\underline{\operatorname{Sex}}$}

Male

$58(48.3 \%)$

Female

$62(51.7 \%)$

$\underline{\text { Age (years) }}$

$\leq 45$

$46(38.4 \%)$

$46-55$

$43(35.8 \%)$

$>55$

$31(25.8 \%)$

$\underline{\text { Education level }}$

Elementary or lower

$73(60.9 \%)$

Secondary or higher

$47(39.1 \%)$

Family income per year (CNY)

$1543.33 \pm 464.49$

Months since the spouse's death (months) $\quad 22.27 \pm 7.59$ (6-34)

US\$1 = CNY 6.36 
Table 2. Hierarchical linear regression models predicting intensity of grief symptoms

\begin{tabular}{lcc}
\hline & Standardized Beta (se) & $p$ \\
\hline Quality of dying of the deceased & $.05(.58)$ & .61 \\
Negative conceptions of death from AIDS & $.19(.20)$ & .04 \\
Death attitudes of the bereaved participants & $-.09(1.40)$ & .47 \\
Approach acceptance & $.40(1.35)$ & $<.001$ \\
Fear of death & $-.07(1.15)$ & .43 \\
Death avoidance & $.14(1.63)$ & .30 \\
Escape acceptance & $.09(1.22)$ & .39 \\
Personal acceptance & $-.29(.06)$ & .003 \\
Resilience
\end{tabular}

These results control for sex, age, education, family income, and time since bereavement. 